\title{
A sensitive detection method for capacitive ultrasonic transducers
}

\author{
A. S. Ergun a) and A. Atalar \\ Department of Electrical and Electronics Engineering, Bilkent University, Bilkent, Ankara, 06533 Turkey \\ B. Temelkuran and E. Özbay \\ Department of Physics, Bilkent University, Bilkent, Ankara, 06533 Turkey
}

(Received 20 February 1998; accepted for publication 6 April 1998)

\begin{abstract}
We report a sensitive detection method for capacitive ultrasonic transducers. Detection experiments at $1.6 \mathrm{MHz}$ reveal a minimum detectable displacement around $2.5 \times 10^{-4} \AA / \sqrt{\mathrm{Hz}}$. The devices are fabricated on silicon using surface micromachining techniques. We made use of microwave circuit considerations to obtain a good displacement sensitivity. Our method also eliminates the dependence of the sensitivity on the ultrasound frequency, allowing the method to be used at low audio frequency and static displacement sensing applications. (C) 1998 American Institute of Physics. [S0003-6951(98)00923-1]
\end{abstract}

Noncontact air-coupled ultrasonic measurements and nondestructive evaluation are becoming more attractive with the development of high frequency, high efficiency, and sensitive transducers. ${ }^{1}$ The basic advantages of air-coupled ultrasonic measurements are their noncontact nature and the short wavelengths of ultrasound in air. Conventional piezoelectric transducers have very large acoustic impedances (in the order of $10^{7} \mathrm{~kg} / \mathrm{m}^{2} \mathrm{~s}$ ) compared to the acoustic impedance of air $\left(400 \mathrm{~kg} / \mathrm{m}^{2} \mathrm{~s}\right)$. This large impedance mismatch at the transducer-air interface introduces an enormous transmission loss, and decreases the coupling efficiency both in generation and detection of ultrasound. To overcome this mismatch, special matching layers can be used. ${ }^{2-4}$ Although the use of matching layers solves the problem to some extent, this technique introduces other problems. Together with air backing, the increase in the coupling efficiency comes at the expense of a narrower bandwidth, and a limited high frequency performance. ${ }^{8}$ Furthermore, the complexity introduced in the production process decreases the reliability, increases the cost, and makes the fabrication of transducer arrays very difficult.

In the past few years silicon capacitive micromachined ultrasonic transducers became an alternative to piezoelectric transducers. ${ }^{5,6}$ Using the standard silicon processes developed in the past 30 years, along with micromachining technology, scientists developed reliable, small, and cheap transducers and transducer arrays with comparable performance. ${ }^{7-9}$ These capacitive transducers usually consist of many circular membranes in parallel (see Refs. 6 and 8 for details), and are used for both generation and detection of ultrasound. The detection depends on the vibration of the membranes due to an incident ultrasound signal. The displacement of the membranes results in a capacitance change which is measured by monitoring the current under a constant bias voltage. The magnitude of the current resulting from $n$ parallel capacitors can be expressed as,

$$
I=\omega_{1} V_{\mathrm{dc}} n C \frac{\Delta x}{x_{0}}
$$

${ }^{a)}$ Electronic mail: sanli@ee.bilkent.edu.tr where $\omega_{1}$ is the ultrasound frequency, $V_{\mathrm{dc}}$ is the bias voltage, $C$ is the capacitance of a single membrane, $x_{0}$ is the electrode spacing of the capacitors, and $\Delta x$ is the displacement. The problems of this method are the large capacitance value needed to achieve reasonable sensitivity, and the dependence of the output on the ultrasound frequency. The former problem is a handicap in building an array of transducers. In this letter we propose an alternative method to measure the displacement in a more sensitive manner.

The new method involves the construction of an artificial transmission line using the membranes as capacitors. An $n$-section artificial transmission line consists of $n$ shunt capacitors $(C)$ linked through inductors $(L)$, as in the circuit representation of an ordinary uniform transmission line. Disregarding the losses, the characteristic impedance $\left(Z_{a}\right)$, and the phase length $\left(\Phi_{0}\right)$ are given by $Z_{a}=\sqrt{L / C}$, and $\Phi_{0}$ $=n \omega_{0} \sqrt{L C}$, where $\omega_{0}$ is the radio frequency $(\mathrm{rf})$.

A capacitance change results in a change in the characteristic impedance and the phase length of the artificial transmission line. For small capacitance variations the change in the characteristic impedance is negligible, whereas the change in the phase length can be significant depending on $n$ and $\omega_{0}$. Note that, using a single section with $n C$ and $n L$ values rather than using $n$ sections with $L$ and $C$ values does not make any difference in terms of the phase length, but decreases the cutoff frequency. A very small capacitance change can be detected by measuring the phase length at a high $\mathrm{rf}$ frequency. An incident ultrasound signal of frequency $\omega_{1}$ vibrates the top plates of the capacitors, and changes the capacitance values. This results in the phase modulation of the rf signal transmitted through the artificial line. For small capacitance variations, the power spectrum of the phase modulation is equivalent to the power spectrum of an amplitude modulation. Therefore, the spectrum of the transmitted signal contains a main signal at $\omega_{0}$, and sidebands at $\omega_{0}$ $\pm \omega_{1}$. In the frequency domain the sidebands are separated from the main signal by an amount equal to the ultrasound frequency. Thus, the ultrasound signal that vibrates the top plates of the capacitors can be extracted by down converting the output of the artificial transmission line as shown in Fig. 1. The magnitude of the output current can be expressed as, 


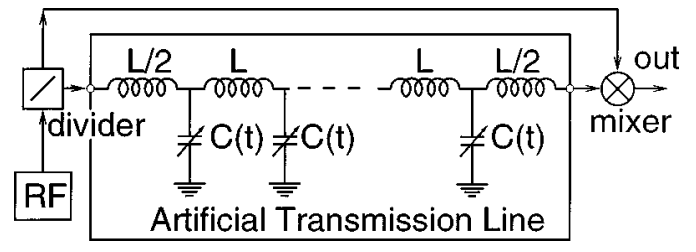

FIG. 1. Our detection system: The phase-modulated rf signal is down converted to obtain the ultrasound signal.

$$
I=G \omega_{0} V_{\mathrm{rf}} n C \frac{\Delta x}{x_{0}}
$$

where $G$ is the conversion gain of the mixer $(\sim 0.5)$, and $V_{\mathrm{rf}}$ is the rf voltage.

Comparison of Eqs. (1) and (2) shows the difference of our method clearly. The large dc bias voltage (in the order of $50 \mathrm{~V}$ ) in the conventional method is replaced by a few volts of $\mathrm{rf}$ amplitude in our method. The reduction in voltage magnitude is compensated with the replacement of the ultrasound frequency $\omega_{1}$ by the rf frequency $\omega_{0}$. Considering an ultrasound frequency in the $\mathrm{MHz}$ range, and a rf signal in the $\mathrm{GHz}$ range, several orders of magnitude improvement in the sensitivity over the conventional method is possible. For applications which involve lower ultrasound frequencies or audio frequencies, the improvement can be even higher.

To verify this method, we fabricated artificial transmission lines on a Si substrate. The capacitors are realized with air bridges, and the inductors are formed with short sections of high impedance transmission lines as shown in Fig. 2(a). The first level metalization defines the inductors and the bottom plates of the capacitors. The air-bridge metalization, which constitutes the top plates of the capacitors, is made of aluminum because of its superior mechanical properties. ${ }^{10}$

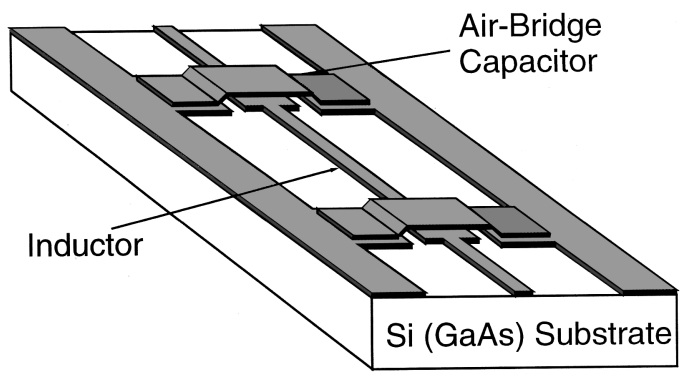

FIG. 2. (a) Schematic, and (b) SEM photograph of an artificial transmission line section.

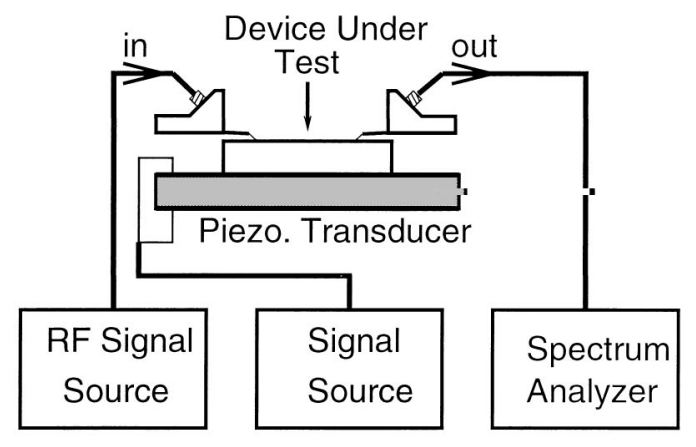

FIG. 3. Detection experiment setup: rf signal source feeds the artificial line, and the signal source drives the piezoelectric transducer. The spectrum analyzer monitors the transmitted signal.

The length and the thickness of the bridges are 50 and $1 \mu \mathrm{m}$, respectively. These values are chosen such that the mechanical resonance frequency of the bridge is $2 \mathrm{MHz} .^{11}$ The sacrificial layer that separates the air bridge from the first metalization is a $1 \mu \mathrm{m}$-thick photoresist baked at $140{ }^{\circ} \mathrm{C}$ for 20 min. This layer is later removed during the lift-off step of the second metalization. The scanning electron microscope (SEM) photograph in Fig. 2(b) shows an air-bridge capacitor of an artificial transmission line section.

Figure 3 shows the experimental setup used for detection experiments. The sample is fixed on the top of a piezoelectric transducer. The transducer is excited at frequency $\omega_{1}$, and the contacts to the artificial transmission line are made by microwave probes. The transmitted signal is monitored by a spectrum analyzer, and the output is plotted as a function of the ultrasound frequency in Fig. 4. The poles and zeros of the acoustic transducer are clearly seen at $(2 k+1) \times 1.1 \mathrm{MHz}$, which are quite sharp because of the unloaded piezoelectric transducer. The frequency response of our detector is also observable from the plot. Roughly, the $3-\mathrm{dB}$ bandwidth is 9 $\mathrm{MHz}$ with the resonance at $1.6 \mathrm{MHz}$. The input is a $10 \mathrm{~mW}$ rf signal at $200 \mathrm{MHz}$. For this input level, $80 \mathrm{nW}$ output power corresponds to $\sim 100$-nm peak vibration of the bridges. Considering thermal noise power at the output of the device, we see that the minimum detectable vibration is $\sim 2.5 \times 10^{-4} \AA / \sqrt{\mathrm{Hz}}$. That is, for a typical system bandwidth of $10 \mathrm{kHz}$, a displacement of $0.025 \AA$ is detectable. The output signal is proportional to the rf frequency $\left(\omega_{0}\right)$, and inversely proportional to the parallel plate separation $\left(x_{0}\right)$. If we increase the rf frequency, and decrease the electrode spacing we can further enhance the sensitivity of our detector

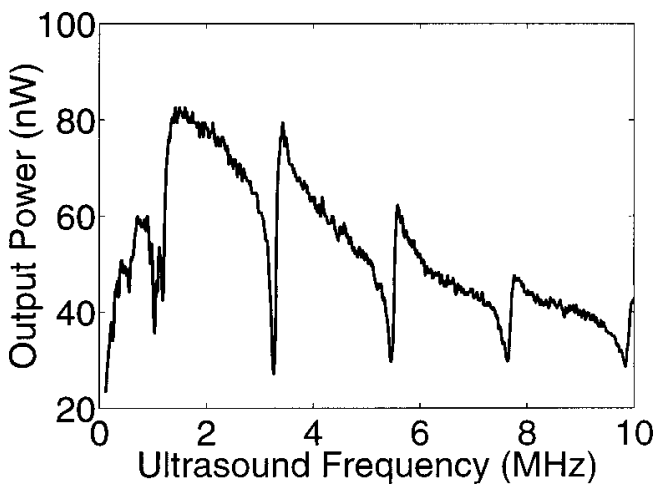

FIG. 4. Frequency response of the device under test. 
by two orders of magnitude. Unfortunately, the poor resistivity of the silicon substrate does not allow the use of very high frequencies. Use of a high resistivity GaAs substrate with a much lower rf loss will make higher microwave frequencies feasible with a corresponding increase in sensitivity. We note that oscillators and mixers can be realized up to several $\mathrm{GHz}$ on silicon substrates and several tens of $\mathrm{GHz}$ on GaAs substrates. It should be possible to fabricate a large array of sensitive detectors monolithically integrated with their associated electronics.

To summarize, a highly sensitive detection method for capacitive ultrasonic transducers is demonstrated. The method eliminates the dependency of the output signal on the ultrasound frequency. Thus, we can apply this method to audio frequency and static displacement sensing applications equally well.

The authors would like to acknowledge the valuable discussions and help of Erhan P. Ata and Ayhan Bozkurt.
${ }^{1}$ D. A. Hutchins and D. W. Schindel, Ultrason. Symp. Proc. IEEE (New York) 2, 1245 (1994).

${ }^{2}$ L. C. Lynnworth, IEEE Trans. Sonics Ultrason. SU-12, 37 (1965).

${ }^{3}$ S. Schiller C. K. Hsieh, C. H. Chou, and B. T. Khuri-Yakub, Rev. Prog. Quant. Nondestr. Eval. 9, 795 (1990).

${ }^{4}$ M. I. Haller and B. T. Khuri-Yakub, Ultrason. Symp. Proc. IEEE (New York) 2, 937 (1992).

${ }^{5}$ M. I. Haller and B. T. Khuri-Yakub, Ultrason. Symp. Proc. IEEE (New York) 2, 1241 (1994)

${ }^{6}$ D. W. Schindel, D. A. Hutchins, L. Zou, and M. Sayer, IEEE Trans. Ultrason. Ferroelectr. Freq. Control 42, 42 (1995).

${ }^{7}$ D. W. Schindel and D. A. Hutchins IEEE Trans. Ultrason. Ferroelectr. Freq. Control 42, 51 (1995).

${ }^{8}$ I. Ladabaum, B. T. Khuri-Yakub, and D. Spoliansky, Appl. Phys. Lett. 68, 7 (1996).

${ }^{9}$ H. T. Soh, I. Ladabaum, A. Atalar, C. F. Quate, and B. T. Khuri-Yakub, Appl. Phys. Lett. 69, 3674 (1996).

${ }^{10}$ C. Goldsmith, J. Randall, S. Eshelman, T. H. Lin, D. Denninston, S. Chen, and B. Norvell, IEEE MTT-S Dig. 2, 1141 (1996).

${ }^{11}$ P. M. Morse and K. U. Ingard, Theoretical Acoustics (Princeton University Press, Princeton, NJ, 1968), p. 175. 